\title{
Offsets, Conchoids and Pedal Surfaces
}

\author{
Martin Peternell, Lukas Gotthart, Juana Sendra and J. Rafael \\ Sendra
}

\begin{abstract}
We discuss three geometric constructions and their relations, namely the offset, the conchoid and the pedal construction. The offset surface $F_{d}$ of a given surface $F$ is the set of points at fixed normal distance $d$ of $F$. The conchoid surface $G_{d}$ of a given surface $G$ is obtained by increasing the radius function by $d$ with respect to a given reference point $O$. There is a nice relation between offsets and conchoids: The pedal surfaces of a family of offset surfaces are a family of conchoid surfaces. Since this relation is birational, a family of rational offset surfaces corresponds to a family of rational conchoid surfaces and vice versa.

We present theoretical principles of this mapping and apply it to ruled surfaces and quadrics. Since these surfaces have rational offsets and conchoids, their pedal and inverse pedal surfaces are new classes of rational conchoid surfaces and rational offset surfaces.
\end{abstract}

Mathematics Subject Classification (2010). 51M,51N,53A.

Keywords. offset surfaces, conchoid surfaces, pedal surfaces, inverse pedal surfaces, Darboux and Dupin cyclides.

\section{Introduction}

There is a large variety of contributions dealing with offset constructions discussing different aspects, see e.g. [2], [4], [5], [10], [11], [16], [21] and references on the topic in [19]. Conchoidal constructions, although not so extensively studied, have been recently addressed by different authors, see for instance [1], [12], [17], [18]. Both constructions were already used in the past: Leibnitz studied parallel curves and ancient Greeks used conchoids. Nowadays they are also used in practical applications: For offsets see for instance [4], [5], [10]. For conchoids see for instance [8], [20], [22].

The offset and the conchoid construction relate to each other via the pedal-point or foot-point construction. Pedal curves were already treated in classical books. For instance [9] describes the algebraic and geometric relationships between a curve and its pedal. Moreover, results connecting offset curves and conchoid curves via pedals are found in the classical literature 
(for instance [7], p. 179). We revisit these classical ideas, provide theoretical principles and extend these results to surfaces.

The foot-point map is a quadratic birational transformation and maps a family of offset surfaces to a family of conchoid surfaces. The inverse map operates analogously. The construction is illustrated at hand of the offset and conchoid surfaces of ruled surfaces and quadrics. Since rational ruled surfaces and quadrics have rational offsets and rational conchoid surfaces, the footpoint map and its inverse generate new families of surfaces having rational conchoids and offsets, respectively. The foot-point map gives further insight to geometric properties of the resulting surfaces, for example that the pedal surfaces of quadrics are Darboux cyclides. Rational universal parameterizations of rational offset and conchoid surfaces are provided.

Notation. Points in Euclidean space $\mathbb{R}^{3}$ are identified with vectors $\mathbf{x} \in \mathbb{R}^{3}$, and $\mathbf{x} \cdot \mathbf{y}$ denotes the scalar product of two vectors $\mathbf{x}, \mathbf{y} \in \mathbb{R}^{3}$. Vectors are considered as column vectors, in particular for matrix-vector products (e.g. $A$. $\mathbf{x}$, for $A \in \mathbb{R}^{3 \times 3}$ and $\mathbf{x} \in \mathbb{R}^{3}$ ). For easier notation and if there is no ambiguity, we denote vectors coordinate-wise as row-vectors and omit the symbol ${ }^{T}$ for transposition. We use the notation $\mathbf{x}^{2}=\|\mathbf{x}\|^{2}$ and analogously for even powers of the norm. The set of points and planes of $\mathbb{R}^{3}$ are denoted by $\mathcal{P}$ and $\mathcal{E}$, respectively.

The projective extension of the Euclidean space $\mathbb{R}^{3}$ is denoted as $\mathbb{P}^{3}$. Points $X \in \mathbb{P}^{3}$ are identified with their homogeneous coordinate vectors $X=\left(x_{0}, x_{1}, x_{2}, x_{3}\right) \mathbb{R}=\left(x_{0}: x_{1}: x_{2}: x_{3}\right)$. A plane $U$ in $\mathbb{P}^{3}$ is the zero-set of a linear equation $U: u_{0} x_{0}+\ldots+u_{3} x_{3}=0$. The coefficients $\left(u_{0}, \ldots, u_{3}\right)$ are the homogeneous coordinates of $U$. To distinguish points from planes, we use $\left(x_{0}, \ldots, x_{3}\right) \mathbb{R}$ for points and $\mathbb{R}\left(u_{0}, \ldots, u_{3}\right)$ for planes. A plane $U \subset \mathbb{P}^{3}$ is identified with a point in the dual space $\mathbb{P}^{3^{\star}}$ of $\mathbb{P}^{3}$. Let $\omega=\mathbb{P}^{3} \backslash \mathbb{R}^{3}$ be the ideal plane in $\mathbb{P}^{3}$, that is $\omega: x_{0}=0$. Ideal points are represented by $(0, \mathbf{x}) \mathbb{R}$, with $\mathbf{x} \in \mathbb{R}^{3}$. For points $X \notin \omega$, the relation between Cartesian coordinates $\mathbf{x}=(x, y, z)$ and homogeneous coordinates is given by

$$
x=\frac{x_{1}}{x_{0}}, y=\frac{x_{2}}{x_{0}}, z=\frac{x_{3}}{x_{0}} .
$$

\section{Geometric Constructions}

Offset Surfaces. Consider a smooth surface $F \subset \mathbb{R}^{3}$ with parametrization $\mathbf{f}(u, v)$ and associated oriented unit normal vector $\mathbf{n}(u, v)$. The one-sided offset surface $F_{d}$ of $F$ at the oriented distance $d \in \mathbb{R}$ admits the parametrization

$$
\mathbf{f}_{d}(u, v)=\mathbf{f}(u, v)+d \mathbf{n}(u, v), \text { with }\|\mathbf{n}\|=1 .
$$

The tangent planes of $F$ and $F_{d}$ at corresponding points $\mathbf{f}(u, v)$ and $\mathbf{f}_{d}(u, v)$ are parallel. As derived in [13], we consider $F$ and $F_{d}$ as the envelope surfaces of their tangent planes

$$
\begin{aligned}
E(u, v): \mathbf{n}(u, v) \cdot \mathbf{x} & =e(u, v), \text { with } e=\mathbf{f} \cdot \mathbf{n}, \text { and } \\
E_{d}(u, v): \mathbf{n}(u, v) \cdot \mathbf{x} & =e(u, v)+d .
\end{aligned}
$$


Let $\bar{F} \subset \mathbb{P}^{3}$ be the projective surface corresponding to $F$. The tangent planes $E$ of $\bar{F}$ determine the surface $\bar{F}^{\star} \subset \mathbb{P}^{3^{\star}}$, called the dual surface of $\bar{F}$. Since $\bar{F}$ is uniquely defined by $F$, we do not distinguish between them and denote both $F$. If we want to emphasize that $F$ is considered as family of tangent planes, we denote it by $F^{\star}$.

If $F^{\star}$ is of degree $n$, it can be expressed as the zero set of the polynomial

$$
F^{\star}\left(u_{0}, \mathbf{u}\right)=u_{0}^{n} f_{0}+\ldots+u_{0}^{n-j} f_{j}(\mathbf{u})+\ldots+f_{n}(\mathbf{u}),
$$

where $f_{j}(\mathbf{u})$ are homogeneous polynomials of degree $j$ in $\mathbf{u}=\left(u_{1}, u_{2}, u_{3}\right)$. The ideal plane $\omega: x_{0}=0$ is tangent to $F$, which means that $\omega \in F^{\star}$, if $f_{0}=0$. Furthermore, $\omega$ is an $r$-fold plane of $F^{\star}$, exactly if $f_{0}=\cdots=f_{r-1}=0$, but $f_{r} \neq 0$.

While a parameterization of $F$ in homogeneous point coordinates is $(1, \mathbf{f}) \mathbb{R}$, parameterizations of $F^{\star}$ and its offset surfaces $F_{d}^{\star}$ read $E=\mathbb{R}(-e, \mathbf{n})$, and $E_{d}=\mathbb{R}(-e-d, \mathbf{n})$. Let $S^{2}:\|\mathbf{x}\|^{2}=1$ be the unit sphere in $\mathbb{R}^{3}$. To make the relations between the offsets and conchoids obvious, we define the map

$$
\begin{aligned}
\varphi: S^{2} \times \mathbb{R} & \rightarrow \mathcal{E} \\
(\mathbf{n}, e) & \mapsto E: \mathbf{n} \cdot \mathbf{x}=e, \text { with } \mathbf{n} \in S^{2}, e \in \mathbb{R} .
\end{aligned}
$$

The offset map $o^{\star}: \mathcal{E} \rightarrow \mathcal{E}$ with respect to the offset distance $d$ is defined by

$$
o^{\star}(E)=o^{\star}(\varphi(\mathbf{n}, e))=\varphi(\mathbf{n}, e+d)=E_{d} .
$$

Focusing on rational surfaces we formulate

Definition 1. A rational surface $F \subset \mathbb{R}^{3}$ is called rational offset surface if $F$ admits a rational parametrization $\mathbf{f}(u, v)$ with rational unit normal vector $\mathbf{n}(u, v)$.

Any rational parametrization $\mathbf{n}(u, v)$ of $S^{2}$ and any rational radius function $e(u, v)$ define a rational offset surface $F$ in that sense. Rational offset surfaces are also called surfaces with Pythagorean normal vectors, abbreviated by $P N$ surfaces. This expresses the fact that there exist polynomials $a(u, v), b(u, v), c(u, v)$ and $d(u, v)$ forming a Pythagorean quadruple, meaning that $a^{2}+b^{2}+c^{2}=d^{2}$. Consequently, $(a, b, c) / d$ is a rational unit normal vector parametrizing $S^{2}$.

An offset surface of $F$ can also be defined as the envelope of a family of spheres of radius $d$, which are centered at $F$. We denote this offset by $O_{d}(F)$, since this definition obviously differs from (1). The geometric properties and relations between $O_{d}(F)$ and $F_{d}$ are studied in [16], and in any case for reducible or irreducible offsets $O_{d}(F)=F_{d} \cup F_{-d}$ holds.

Remark 1. The notation rational offset surface expresses that $F_{d}$ and $O_{d}(F)$ admit rational parameterizations, but does not imply that $F_{d}$ or $O_{d}(F)$ is rational in the sense of algebraic geometry. For instance, the offset surfaces of regular quadrics $\subset \mathbb{R}^{3}$ admit real rational parametrizations, but only the offsets of spheres and paraboloids are rational surfaces and offsets of ellipsoids and hyperboloids are unirational in that sense. 
Conchoid Surfaces. Consider a smooth surface $G \subset \mathbb{R}^{3}, d \in \mathbb{R}$, and a fixed reference point $O$, always assumed to be the origin of a Cartesian coordinate system. Let $G$ be represented by a polar representation $\mathbf{g}(u, v)=r(u, v) \mathbf{s}(u, v)$, with $\|\mathbf{s}(u, v)\|=1$. We call $\mathbf{s}(u, v)$ the spherical part of $\mathbf{g}(u, v)$ and $r(u, v)$ its radius function. The one-sided conchoid surface $G_{d}$ of $G$ is obtained by increasing the radius function $r(u, v)$ by $d$ and thus $G_{d}$ admits the polar representation

$$
\mathbf{g}_{d}(u, v)=(r(u, v)+d) \mathbf{s}(u, v) .
$$

In a similar manner to (4), we define the map

$$
\begin{aligned}
\gamma: S^{2} \times \mathbb{R} & \rightarrow \mathcal{P} \\
(\mathbf{s}, r) & \mapsto \mathbf{g}=r \mathbf{s}, \text { with } \mathbf{s} \in S^{2}, r \in \mathbb{R} .
\end{aligned}
$$

The conchoidal map $c: \mathcal{P} \rightarrow \mathcal{P}$, specifying the relation between a surface $G$ and its conchoid surface $G_{d}$, is defined as

$$
c(\gamma(\mathbf{s}, r))=\gamma(\mathbf{s}, r+d) .
$$

Similarly to the offsets $F_{d}$ and $O_{d}(F)$, a conchoid surface of $G$ can be defined as the set of points $Q$ in the line $O P$ at distance $d$ of a moving point $P \in G$. We denote this surface by $C_{d}(G)$, and obviously this differs from $G_{d}$, but in any case we have $C_{d}(G)=G_{d} \cup G_{-d}$. For more details on these constructions see [1], [17] and [18]. Focusing on rational surfaces we define

Definition 2. A surface $G$ is called rational conchoid surface with respect to the reference point $O$ if $G$ admits a rational polar representation $r(u, v) \mathbf{s}(u, v)$, with a rational radius function $r(u, v)$ and a rational parametrization $\mathbf{s}(u, v)$ of the unit sphere $S^{2}$.

If $G$ is a rational conchoid surface, $G_{d}$ admits the rational representation (6). A similar statement to Remark 1 is also valid for conchoid surfaces. Pedal Surfaces. The foot-point map $\alpha: \mathcal{E} \rightarrow \mathcal{P}$ with respect to the reference point $O$ transforms planes $E$ to points $P$, see Figure 1(a). It is rational and bijective except for planes $E$ passing through $O$. The inverse map, denoted by $\alpha^{\star}: \mathcal{P} \backslash\{O\} \rightarrow \mathcal{E}$, transforms points $P \neq O$ to planes $E \ni P$ which have $O P$ as normal. These maps read

$$
\begin{array}{lll}
\alpha: \quad E: \mathbf{x} \cdot \mathbf{n}=e & \mapsto \alpha(E): P=\frac{e}{\|\mathbf{n}\|^{2}} \mathbf{n}, \\
\alpha^{\star}: \quad P=\mathbf{p} & \mapsto \alpha^{\star}(P)=E: \mathbf{x} \cdot \mathbf{p}=\mathbf{p} \cdot \mathbf{p} .
\end{array}
$$

The quadratic maps $\alpha$ and $\alpha^{\star}$ are the basic ingredients to construct rational conchoid surfaces from rational offset surfaces and vice versa.

Definition 3. Consider a surface $F$, its dual surface $F^{\star}$ and a fixed reference point $O$. The surface $G=\alpha\left(F^{\star}\right)$ is called the pedal surface of $F$, and consists of the foot-points of the tangent planes of $F$ with respect to $O$. Conversely, the surface $F^{\star}=\alpha^{\star}(G)$ is called the negative or inverse pedal surface of $G$ with respect to $O$. 


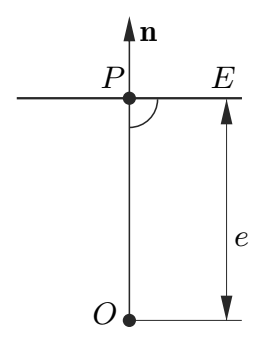

(a) Foot-point map

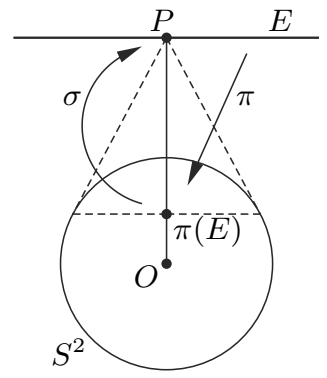

(b) $\alpha=\sigma \circ \pi$

FiguRE 1. Geometric properties of the foot-point map $\alpha$

To get more insight to the correspondence between offsets and conchoids we write $\alpha$ and $\alpha^{\star}$ in terms of homogeneous coordinates. The map $\alpha: \mathcal{E} \rightarrow \mathcal{P}$ uniquely determines the map $\bar{\alpha}: \mathbb{P}^{3^{\star}} \rightarrow \mathbb{P}^{3}$, and thus both maps are denoted by $\alpha$; analogously for $\alpha^{\star}$. Using the abbreviations $\mathbf{x}=\left(x_{1}, x_{2}, x_{3}\right)$ and $\mathbf{u}=$ $\left(u_{1}, u_{2}, u_{3}\right)$, both maps read

$$
\begin{array}{ll}
\alpha: U=\mathbb{R}\left(u_{0}, \mathbf{u}\right) & \mapsto \quad X=\left(x_{0}, \mathbf{x}\right) \mathbb{R}=\left(-\mathbf{u}^{2}, u_{0} \mathbf{u}\right) \mathbb{R}, \\
\alpha^{\star}: X=\left(x_{0}, \mathbf{x}\right) \mathbb{R} & \mapsto \quad U=\mathbb{R}\left(u_{0}, \mathbf{u}\right)=\mathbb{R}\left(-\mathbf{x}^{2}, x_{0} \mathbf{x}\right) .
\end{array}
$$

The exceptional planes of $\alpha$ are $\omega: x_{0}=0$ and the tangent planes of the isotropic cone $\Delta: u_{0}=0, \mathbf{u}^{2}=0$ with vertex $O$. The exceptional points of $\alpha^{\star}$ are $O$ and the points of the absolute conic $j: x_{0}=0, \mathbf{x}^{2}=0$. Considering the cone $\Delta$ as point set, we have $j=\Delta \cap \omega$. Let $\sigma: \mathbb{P}^{3} \rightarrow \mathbb{P}^{3}$ be the inversion at $S^{2}$, and let $\pi: \mathbb{P}^{3^{\star}} \rightarrow \mathbb{P}^{3}$ be the polarity with respect to $S^{2}$, and $\pi^{\star}: \mathbb{P}^{3} \rightarrow \mathbb{P}^{3^{\star}}$ its dual map. These maps satisfy $\sigma=\sigma^{-1}$ and $\pi \circ \pi^{\star}=\mathrm{id}$, and read

$$
\begin{aligned}
\sigma: X=\left(x_{0}, \ldots, x_{3}\right) \mathbb{R} & \mapsto \sigma(X)=\left(x_{1}^{2}+x_{2}^{2}+x_{3}^{2}, x_{0} x_{1}, x_{0} x_{2}, x_{0} x_{3}\right) \mathbb{R}, \\
\pi: U=\mathbb{R}\left(u_{0}, \ldots, u_{3}\right) & \mapsto \pi(U)=X=\left(-u_{0}, u_{1}, u_{2}, u_{3}\right) \mathbb{R}, \\
\pi^{\star}: X=\left(x_{0}, \ldots, x_{3}\right) \mathbb{R} & \mapsto \pi^{\star}(X)=U=\mathbb{R}\left(-x_{0}, x_{1}, x_{2}, x_{3}\right) .
\end{aligned}
$$

It is easily verified (see Figure $1(\mathrm{~b})$ ) that $\alpha$ and $\alpha^{\star}$ satisfy the relations

$$
\alpha=\sigma \circ \pi, \text { and } \alpha^{\star}=\pi^{\star} \circ \sigma .
$$

\section{The relation between offsets and conchoids}

There is a nice relation between the offsets and the conchoids via the pedal construction, see e.g. [7], pg. 179. We use the parameterizations $\varphi$ and $\gamma$ from equations (4) and (7) and the maps $o^{\star}$ and $c$ from (5) and (8). The representations (10) of $\alpha$ and $\alpha^{\star}$ prove the commutativity of the following diagrams. 

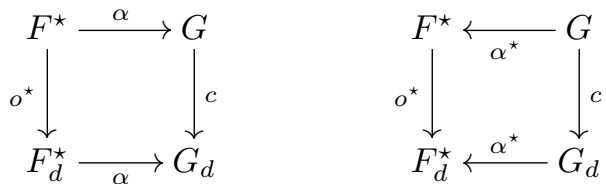

Proposition 4. Let $F_{d}^{\star}$ be offset surfaces of a dual surface $F^{\star}$ at distance $d$. Then the pedal surfaces $G_{d}=\alpha\left(F_{d}^{\star}\right)$ are conchoid surfaces of $G=\alpha\left(F^{\star}\right)$ at distance $d$. Let $G_{d}$ be conchoid surfaces of a surface $G$ at distance $d$. Then $F_{d}^{\star}=\alpha^{\star}\left(G_{d}\right)$ are the offset surfaces of $F^{\star}=\alpha^{\star}(G)$ at distance $d$.

Injectivity. The maps $\varphi$ and $\gamma$ are considered as local parametrizations of the tangent planes or the points of surfaces in $\mathbb{R}^{3}$. Since $\varphi(\mathbf{n}, e)$ and $\varphi(-\mathbf{n},-e)$ map to the same non-oriented plane in $\mathcal{E}$, and $\gamma(\mathbf{s}, r)$ and $\gamma(-\mathbf{s},-r)$ define the same point in $\mathcal{P}$, these maps are not injective globally. If injectivity is an issue, one can identify antipodal points in $S^{2} \times \mathbb{R}$ or may define an orientation of a plane with help of $\mathbf{n}$. However, an orientation of a point sounds weird.

The definition of the one-sided offset $F_{d}$ in (1) uses an oriented normal vector field. But the offset surface $O_{d}(F)$ of an algebraic surface $F$ contains both, the inner and outer offset. The same holds for the conchoid surface $C_{d}(G)$. In order to parametrize the offsets or conchoids of algebraic surfaces, the base surface is traced twice. Since the foot-point construction ignores orientations, we consider planes in $\mathbb{R}^{3}$ as non-oriented.

Universal parametrizations. Combining universal rational parametrizations of $S^{2}$ with the respective maps $\varphi$ and $\gamma$ results in respective universal parameterizations of offset and conchoid surfaces. Following [3] one chooses four arbitrary polynomials $a(u, v), b(u, v), c(u, v)$ and $d(u, v)$ without common factor. Let $A=2(a c+b d), B=2(b c-a d), C=a^{2}+b^{2}-c^{2}-d^{2}$, and $D=a^{2}+b^{2}+c^{2}+d^{2}$, then

$$
\mathbf{q}(u, v)=\frac{1}{D}(A, B, C)
$$

is a rational parametrization of $S^{2}$. Any rational parameterization of $S^{2}$ is obtained by specifying the functions $a(u, v), \ldots, d(u, v)$.

Proposition 5. A universal rational parametrization of a rational offset surface or a rational conchoid surface is of the form $\varphi(\mathbf{q}, \rho)$ or $\gamma(\mathbf{q}, \rho)$, respectively, with a rational function $\rho(u, v)$.

The maps $\alpha$ and $\alpha^{\star}$ transfer rational parametrizations of offset surfaces to conchoid surfaces and vice-versa. The implicit representations of $\alpha$ and $\alpha^{\star}$ show the relations between the degrees of $F^{\star}$ and $G=\alpha\left(F^{\star}\right)$. Let $F^{\star}$ be given by (3). Inserting (10) into $F^{\star}$ yields

$$
\widetilde{G}\left(x_{0}, \mathbf{x}\right)=\left(-\mathbf{x}^{2}\right)^{n} f_{0}+\ldots+\left(-\mathbf{x}^{2}\right)^{n-k} x_{0}^{k} f_{k}(\mathbf{x})+\ldots+x_{0}^{n} f_{n}(\mathbf{x}) .
$$

The polynomial $\widetilde{G}$ might have factors $x_{0}^{r}$ or $\mathbf{x}^{2 k}$, which are split off. The pedal surface $G=\alpha\left(F^{\star}\right)$ is thus defined by the remaining irreducible component of $\widetilde{G}$. If $f_{0}=\ldots f_{r-1}=0$, but $f_{r} \neq 0$, then $\widetilde{G}$ contains the factor $x_{0}^{r}$. If 
$f_{n}$ has the factor $\mathbf{u}^{2}$, it also appears in $\widetilde{G}$. More generally, assume that the polynomials $f_{n}, \ldots, f_{n-k}$ have a factor of the form

$$
f_{n-j}(\mathbf{u})=\left(\mathbf{u}^{2}\right)^{k-j} h_{n-2 k+j}(\mathbf{u}), \text { for } j=0, \ldots, k,
$$

with $\operatorname{gcd}\left(h_{n-2 k+j}, \mathbf{u}^{2}\right)=1$. Then the polynomial (14) has the factor $\mathbf{x}^{2 k}$, and the irreducible component $G$ is of degree $2 n-2 k$. We have the following result.

Proposition 6. Let $F^{\star}$ be of degree $n$, then the degree of $G$ is $2 n-r-2 k$, with $r$ as the multiplicity of $\omega$ and $k$ as the multiplicity of the cone $u_{0}=0, \mathbf{u}^{2}=0$.

Analogously one computes the polynomial of a surface $F^{\star}=\alpha^{\star}(G)$. One exchanges $F^{\star}$ by $G$, dual coordinates $u_{i}$ by $x_{i}$, the ideal plane $\omega$ by $O$ and the isotropic cone $\Delta \ni O$ by the conic $j \subset \omega$. We continue with two elementary examples. The first discusses conchoid surfaces of a plane and their inverse pedal surfaces. The second example considers offsets of a sphere and their pedal surfaces.

Conchoid surfaces of a plane. Consider the plane $G: z=1$ and the reference point $O=(0,0,0)$. Figure 2(a) illustrates the 2d-case. Using homogeneous coordinates, the plane $G$ and its conchoid surfaces $G_{d}$ are represented by

$$
G(X)=x_{3}-x_{0}, G_{d}(X)=d^{2} x_{0}^{2} x_{3}^{2}-\left(x_{1}^{2}+x_{2}^{2}+x_{3}^{2}\right) G(X)^{2} .
$$

Since the highest power of $x_{0}$ is two, $O$ is a double point of $G_{d}$, and $\alpha^{\star}\left(G_{d}\right)$ has the factor $u_{0}^{2}$. Dividing by $\left(u_{1}^{2}+u_{2}^{2}+u_{3}^{2}\right)$ gives the polynomials determining the paraboloid $F^{\star}=\alpha(G)$ and its offsets $F_{d}^{\star}=\alpha\left(G_{d}\right)$,

$$
F^{\star}(U)=u_{1}^{2}+u_{2}^{2}+u_{3}^{2}+u_{0} u_{3}, F_{d}^{\star}(U)=d^{2} u_{3}^{2}\left(u_{1}^{2}+u_{2}^{2}+u_{3}^{2}\right)-F^{\star}(U)^{2} .
$$

Offsets of a sphere. Consider the sphere $F:(x-m)^{2}+y^{2}+z^{2}-R^{2}=0$ and its offset spheres $F_{d}$. Figure $2(\mathrm{~b})$ shows the 2 d-case. We represent them as dual surfaces by

$$
F_{d}^{\star}(U)=\left((R+d)^{2}-m^{2}\right) u_{1}^{2}+(R+d)^{2}\left(u_{2}^{2}+u_{3}^{2}\right)-2 m u_{0} u_{1}-u_{0}^{2} .
$$

The map $\alpha$ transforms $F_{d}^{\star}$ to a family of conchoid surfaces

$G_{d}(X)=x_{0}^{2}\left(x_{1}^{2}\left((R+d)^{2}-m^{2}\right)+(R+d)^{2}\left(x_{2}^{2}+x_{3}^{2}\right)\right)+2 m x_{0} x_{1} \mathbf{x}^{2}-\mathbf{x}^{4}$,

where $\mathbf{x}=\left(x_{1}, x_{2}, x_{3}\right)$ and $\mathbf{x}^{4}=\left(\mathbf{x}^{2}\right)^{2}$. Since the highest power of $x_{0}$ is two, $O$ is a double point of $G_{d}$. When letting $R=0$ and $d=0$, we have $F^{\star}(U)=\left(u_{0}+m u_{1}\right)^{2}$, a two-fold bundle of planes through $M=(m, 0,0)$. $F^{\star}(U)=u_{0}+m u_{1}$ and $F_{d}^{\star}$ are mapped by $\alpha$ to the sphere $G(X)=\mathbf{x}^{2}-m x_{0} x_{1}$, with diameter $O M$, and its conchoid surfaces $G_{d}$.

\section{Rational ruled surfaces and regular quadrics}

We derive geometric properties of pedal and inverse pedal surfaces of ruled surfaces and quadrics. These surfaces are rational offset surfaces and rational conchoid surfaces, see [11] and [12]. The maps $\alpha$ and $\alpha^{\star}$ preserve these properties and generate new families of these surfaces classes. 


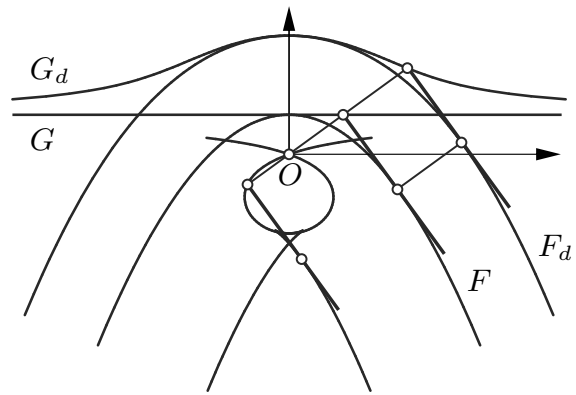

(a) Conchoid $G_{d}$ of a line $G$ and offset $F_{d}$ of a parabola $F$

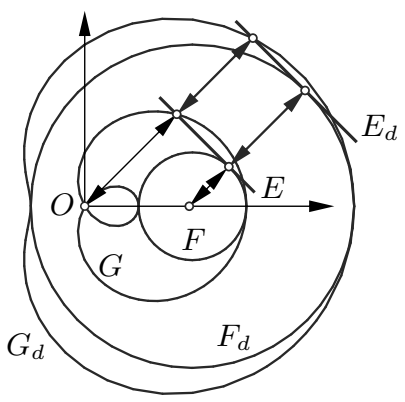

(b) Offset $F_{d}$ of the circle $F$ and conchoid $G_{d}$ of the limason $G$

FiguRE 2. Relation between offsets and conchoids

\subsection{Pedal surfaces of rational ruled surfaces}

Consider a rational ruled surface $F^{\star}$ as dual surface. Any ruling $l \subset F^{\star}$ is a pencil of planes. Let $D$ be the foot-point of $O$ on $l$. According to Thales' theorem, the pedal curve $\alpha(l)$ is a circle with diameter $O D$, in a plane perpendicular to $l$, see Figure 3(a). Thus, the pedal surface $G=\alpha\left(F^{\star}\right)$ contains a one-parameter family of circles in planes perpendicular to the lines $l$.

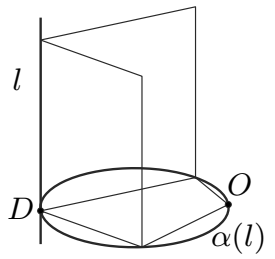

(a) Pedal of a pencil $l$

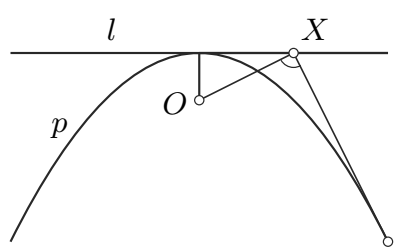

(b) Inverse pedal of a line $l$

Figure 3. Pedal and inverse pedal curve of a line

A rational polar representation of $G=\alpha\left(F^{\star}\right)$ is derived from a rational offset representation of $F^{\star}$. Consider an affine parameterization $\mathbf{f}(u, v)=$ $\mathbf{c}(u)+v \mathbf{e}(u)$ of $F$, and their partial derivatives $\mathbf{f}_{u}=\dot{\mathbf{c}}+v \dot{\mathbf{e}}$ and $\mathbf{f}_{v}=\mathbf{e}$. The normal vector $\mathbf{n}$ of $\mathbf{f}$ reads $\mathbf{n}(u, v)=\mathbf{n}_{1}(u)+v \mathbf{n}_{2}(u)$, with $\mathbf{n}_{1}=\dot{\mathbf{c}} \times \mathbf{e}$, and $\mathbf{n}_{2}$ $=\dot{\mathbf{e}} \times \mathbf{e}$. In case that $\mathbf{n}_{1}(u)$ and $\mathbf{n}_{2}(u)$ are linearly dependent for all $u, F$ is a developable ruled surface. Since $F$ is the envelope of a one-parameter family of planes, $G$ degenerates to a curve.

Let $\mathbf{n}_{1}, \mathbf{n}_{2}$ be linearly independent. We intend to construct a rational unit normal vector field of $F$, and we require $\|\mathbf{n}\|^{2}=w^{2}$, with some rational function $w(u, v)$ to be determined. To simplify the computation, $\mathbf{c}(u)$ is replaced by the striction curve $\mathbf{s}(u)=\mathbf{c}(u)+v_{s}(u) \mathbf{e}(u)$. The normal vector $\mathbf{n}_{s}$ at $\mathbf{s}$ satisfies $\mathbf{n}_{s} \cdot \mathbf{n}_{2}=0$. This relation determines the parameter $v_{s}(u)=$ $-[(\dot{\mathbf{c}} \times \mathbf{e}) \cdot(\dot{\mathbf{e}} \times \mathbf{e})] /(\dot{\mathbf{e}} \times \mathbf{e})^{2}$. We represent $F$ by $\mathbf{f}(u, v)=\mathbf{s}(u)+v \mathbf{e}(u)$. The 
condition $\|\mathbf{n}\|^{2}=w^{2}$ turns into $\mathbf{n}_{s}^{2}+v^{2} \mathbf{n}_{2}^{2}-w^{2}=0$, with affine coordinates $v$ and $w$. Substituting $v=y_{2} / y_{1}$ and $w=y_{0} / y_{1}$, yields a real rational family of conics in $\mathbb{P}^{2}$,

$a(u): a_{1}(u) y_{1}^{2}+a_{2}(u) y_{2}^{2}-y_{0}^{2}=0$, with $a_{1}(u)=\mathbf{n}_{s}(u)^{2}, a_{2}(u)=\mathbf{n}_{2}(u)^{2}$.

There exist rational parameterizations $\mathbf{y}(u, t)=\left(y_{0}, y_{1}, y_{2}\right)(u, t)$ (see for instance [15]), in a way that $\mathbf{y}\left(u_{0}, t\right)$ is a parameterization of the conic $a\left(u_{0}\right)$, for any fixed $u_{0} \in \mathbb{R}$. This yields a rational offset parameterization

$$
\begin{aligned}
\mathbf{f}(u, t) & =\mathbf{s}(u)+\frac{y_{2}(u, t)}{y_{1}(u, t)} \mathbf{e}(u), \text { and } \\
\mathbf{n}(u, t) & =\mathbf{n}_{s}(u)+\frac{y_{2}(u, t)}{y_{1}(u, t)} \mathbf{n}_{2}(u) \text { with }\|\mathbf{n}(u, t)\|=\frac{y_{0}(u, t)}{y_{1}(u, t)} .
\end{aligned}
$$

The homogeneous coordinates of the tangent planes of $F$ 's offset surfaces $F_{d}$ are $E_{d}=\mathbb{R}(-\mathbf{f} \cdot \mathbf{n}-d\|\mathbf{n}\|, \mathbf{n})$. Applying $\alpha$ yields $\alpha\left(E_{d}\right)=\left(\mathbf{n}^{2},(\mathbf{f} \cdot \mathbf{n}+d\|\mathbf{n}\|) \mathbf{n}\right) \mathbb{R}$. The corresponding rational polar representation of $G_{d}=\alpha\left(F_{d}^{\star}\right)$ reads

$$
\mathbf{g}_{d}(u, t)=\left(\frac{\mathbf{f} \cdot \mathbf{n}}{\|\mathbf{n}\|}+d\right) \frac{\mathbf{n}}{\|\mathbf{n}\|} .
$$

Theorem 7. The pedal surface $G$ of a non-developable rational ruled surface $F$ is generated by a rational family of circles in planes through $O$ and perpendicular to $F$ 's lines $l$. The pedal surfaces $G_{d}$ of $F$ 's offset surfaces $F_{d}$ are rational conchoid surfaces. The conchoid surfaces $G_{d}$ of $G$ are generated by the planar conchoid curves of the circles lying on $G$.

\subsection{Inverse pedal surfaces of rational ruled surfaces}

Consider a rational ruled surface $G$ as set of points, and the reference point $O$. Since $G$ is a rational conchoid surface (see [12]), its inverse pedal surface $F^{\star}=$ $\alpha^{\star}(G)$ is a rational offset surface. There are no restrictions on $G$ concerning developability, but $G$ might be a tangent surface of a curve, a cylinder, a cone or a plane. The latter case makes sense only if $O \notin G$.

Consider a line $l \subset G$ and points $X \in l$. The one-parameter family of planes $\alpha^{\star}(X)$ envelope a parabolic cylinder $P$, with $O$ as focal point of the cross section parabola $p$ in the plane connecting $O$ and $l$, see Figure 3(b). The vertex of the parabola $p$ is the foot-point of $O$ on $l$. Thus, the inverse pedal surface $F$ of a ruled surface $G$ is the envelope of that family of parabolic cylinders $P$.

We represent $G$ by a rational polar representation $\mathbf{g}(u, v)$ whose norm $\|\mathbf{g}(u, v)\|=w(u, v)$, is a rational function $w(u, v)$, see [12]. Applying $\alpha^{\star}$ maps $\mathbf{g}$ to a family of tangent planes $E:(\mathbf{x}-\mathbf{g}) \cdot \mathbf{g}=0$ whose normal vector $\mathbf{g}$ has rational length $w$. Consequently, $\mathbf{f}(u, t)=E \cap E_{u} \cap E_{v}$ is a rational offset parameterization of $F$, with rational unit normal vector $\mathbf{n}=\mathbf{g} /\|\mathbf{g}\|$.

Theorem 8. The inverse pedal surface $F=\alpha^{\star}(G)$ of a rational ruled surface $G$ is a rational conchoid surface. $F$ is the envelope of a one-parameter family of parabolic cylinders $P(u)$ with cross section parabolas $p(u)$ with common 
focal point $O$. The offset surfaces $F_{d}$ are the envelopes of the offset cylinders $P_{d}(u)$, whose cross sections are the planar offsets of the parabolas $p(u)$.

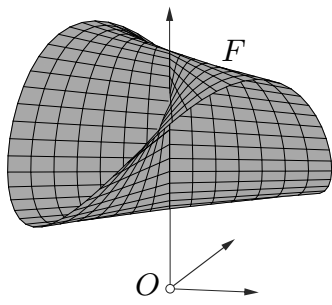

(a) Plücker's conoid $F$

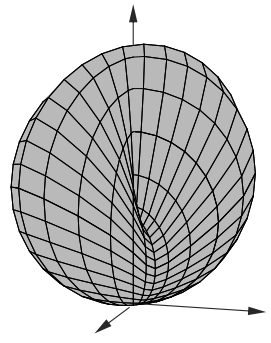

(b) Pedal surface of $F \quad$ (c) Inverse pedal surface of $F$

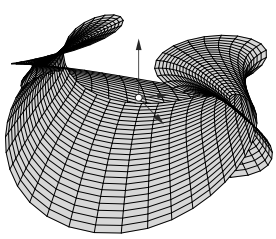

Figure 4. Pedal and inverse pedal surface of Plücker's conoid $\Phi$ with respect to $O$

Pedal surfaces of Plücker's conoid. We consider Plücker's conoid $F$, which is a ruled surface of degree three, projectively equivalent to Whitney's umbrella. A possible parametrization of $F$ reads $\mathbf{f}(r, \varphi)=(r \cos \varphi, r \sin \varphi, \sin 2 \varphi)$, with directrix curve $\mathbf{c}(\varphi)=(0,0, \sin 2 \varphi)$, direction vector $\mathbf{e}(\varphi)=(\cos \varphi, \sin \varphi, 0)$ and normal vector $\mathbf{n}=(-2 \sin \varphi \cos 2 \varphi, 2 \cos \varphi \cos 2 \varphi,-r)$. The point set of $F$ is the zero-set of the polynomial $F(X)=x_{3}\left(x_{1}^{2}+x_{2}^{2}\right)-2 x_{0} x_{1} x_{2}$. For computing the pedal surfaces, we represent $F$ and its offsets $F_{d}$ as dual surfaces

$$
\begin{aligned}
& F^{\star}(U)=u_{0}\left(u_{1}^{2}+u_{2}^{2}\right)-2 u_{1} u_{2} u_{3}, \text { with } U=\left(u_{0}, \ldots, u_{3}\right), \\
& F_{d}^{\star}(U)=d^{2}\left(u_{1}^{2}+u_{2}^{2}\right)^{2}\left(u_{1}^{2}+u_{2}^{2}+u_{3}^{2}\right)-F^{\star}(U)^{2} .
\end{aligned}
$$

The pedal surfaces $G$ and $G_{d}$ of $F$ and $F_{d}$ are rational conchoid surfaces, represented by the respective polynomials

$$
\begin{aligned}
G(X) & =2 x_{0} x_{1} x_{2} x_{3}+\left(x_{1}^{2}+x_{2}^{2}\right)\left(x_{1}^{2}+x_{2}^{2}+x_{3}^{2}\right), \text { and } \\
G_{d}(X) & =d^{2} x_{0}^{2}\left(x_{1}^{2}+x_{2}^{2}\right)^{2}\left(x_{1}^{2}+x_{2}^{2}+x_{3}^{2}\right)-G(X)^{2} .
\end{aligned}
$$

In order to represent $G_{d}$ by a rational polar representation, we start with a rational offset representation of $F$. Thus we have $\|\mathbf{n}\|^{2}=4 \cos ^{2} 2 \varphi+r^{2}=w^{2}$, whose right hand side is a family of conics in $\mathbb{R}^{2}$ with coordinates $r$ and $w$. It can be parameterized directly by

$$
r(\varphi, t)=\frac{2 \cos 2 \varphi \cos t}{\sin t}, \text { and } w(\varphi, t)=\|\mathbf{n}\|=\frac{2 \cos 2 \varphi}{\sin t} .
$$

Plücker's conchoid $F$ admits the rational offset parameterization $\mathbf{f}(r(\varphi, t), \varphi)$, with unit normal vector $\mathbf{n}(\varphi, t)=(-\sin \varphi \sin t, \cos \varphi \sin t, \cos t)$. The tangent planes of $F$ and $F_{d}$ are represented by

$$
E_{d}(\varphi, t)=\mathbb{R}(-\cos t \sin 2 \varphi-d,-\sin \varphi \sin t, \cos \varphi \sin t, \cos t) .
$$

Applying $\alpha$ gives the rational polar representation of $G_{d}$,

$$
\mathbf{g}_{d}=\alpha\left(E_{d}\right)=(\cos t \sin 2 \varphi+d)(-\sin \varphi \sin t, \cos \varphi \sin t, \cos t) .
$$


The line $x_{1}=x_{2}=0$ is a double line of $F$. For the computation of the equations and the parameterizations we choose $O$ as symmetry point on the $z$-axis, since the equations for offsets, pedal surfaces and inverse pedal surfaces are more compact. The illustrations in Figure 4 have been generated for a different reference point on the $z$-axis.

Inverse pedal surfaces of Plücker's conoid. In contrast to the last paragraph, Plücker's conoid $F$ is considered as point set. Let $F$ and its conchoid surfaces $F_{d}$ be the zero-sets of the respective polynomials

$$
\begin{aligned}
A(X) & =x_{3}\left(x_{1}^{2}+x_{2}^{2}\right)-2 x_{0} x_{1} x_{2}, \text { and } \\
A_{d}(X) & =d^{2}\left(x_{1}^{2}+x_{2}^{2}\right)^{2} x_{0}^{2} x_{3}^{2}-\left(x_{1}^{2}+x_{2}^{2}+x_{3}^{2}\right) A(X)^{2} .
\end{aligned}
$$

The inverse foot-point map $\alpha^{\star}$ transforms $F$ and $F_{d}$ to $B^{\star}$ and a family of rational offset surfaces $B_{d}^{\star}$, defined by the respective polynomials

$$
\begin{aligned}
& B^{\star}(U)=u_{0} u_{3}\left(u_{1}^{2}+u_{2}^{2}\right)+2 u_{1} u_{2}\left(u_{1}^{2}+u_{2}^{2}+u_{3}^{2}\right), \\
& B_{d}^{\star}(U)=d^{2}\left(u_{3}^{2}\right)\left(u_{1}^{2}+u_{2}^{2}\right)^{2}\left(u_{1}^{2}+u_{2}^{2}+u_{3}^{2}\right)-B^{\star}(U)^{2} .
\end{aligned}
$$

Since $F$ is a rational conchoid surface, there exists a parameterization $\mathbf{a}(\varphi, v)$ with rational norm. This and the parameterization of its conchoid surfaces read

$$
\begin{aligned}
\mathbf{a}(\varphi, v) & =\left(\frac{2 \sin \varphi \cos v^{2} \sin v}{\cos \varphi}, \frac{2 \sin \varphi \cos v \sin v^{2}}{\cos \varphi}, 2 \cos v \sin v\right), \\
\mathbf{a}_{d}(\varphi, v) & =\mathbf{a}(\varphi, v)+d \frac{\mathbf{a}(\varphi, v)}{\|\mathbf{a}(\varphi, v)\|}, \text { with }\|\mathbf{a}\|=\frac{2 \cos v \sin v}{\cos \varphi} .
\end{aligned}
$$

Rational offset parameterizations of $B^{\star}$ and $B_{d}^{\star}$ can be derived from (17).

\subsection{Pedal surfaces of quadrics}

Consider a quadric $F \subset \mathbb{P}^{3}$ as family of tangent planes. Quadratic cylinders and cones are excluded since their pedal 'surfaces' are curves. The dual surface $F^{\star} \subset \mathbb{P}^{3^{\star}}$ is also a quadric. With $U=\left(u_{0}, \ldots, u_{3}\right)^{T}, F^{\star}$ is the zero-set of the quadratic polynomial

$$
F^{\star}(U)=U^{T} \cdot A \cdot U, \text { with } A \in \mathbb{R}^{4 \times 4} \text {, and } A=A^{T} .
$$

Regular dual quadrics. Consider a regular quadric $F$, and a reference point $O \notin F$. We distinguish the cases where $F$ is a paraboloid $\left(\omega \in F^{\star}\right)$ or $F$ is either a hyperboloid or an ellipsoid. We start with the latter case with $F^{\star}$ expressed by

$$
F^{\star}(U)=a_{0} u_{0}^{2}+a_{1} u_{1}^{2}+a_{2} u_{2}^{2}+a_{3} u_{3}^{2}+u_{0}\left(b_{1} u_{1}+b_{2} u_{2}+b_{3} u_{3}\right) .
$$

Letting $\mathbf{x}=\left(x_{1}, x_{2}, x_{3}\right)$, its pedal surface $G=\alpha\left(F^{\star}\right)$ is the zero set of

$$
G(X)=x_{0}^{2}\left(a_{1} x_{1}^{2}+a_{2} x_{2}^{2}+a_{3} x_{3}^{2}\right)-x_{0} \mathbf{x}^{2}\left(b_{1} x_{1}+b_{2} x_{2}+b_{3} x_{3}\right)+a_{0} \mathbf{x}^{4} .
$$

Since $\pi\left(F^{\star}\right)$ is a quadric, $G=\sigma\left(\pi\left(F^{\star}\right)\right)$ is the image of a quadric with respect to the inversion $\sigma$. The reference point $O$ is a double point of $G$. The pedal surface $G$ is a special Darboux cyclide, see Remark 2, an algebraic surface typically of degree four, whose intersection with $\omega$ is the absolute conic $j$, typically with multiplicity two. We mention that parabolic Darboux cyclides 
are algebraic surfaces of order three, whose intersection with $\omega$ contains, besides $j$, a real line, compare equation (20). We note that not all Darboux cyclides are the images of quadrics with respect to an inversion.

Remark 2. Consider $S^{3}: \mathbf{y}^{T} \cdot \operatorname{diag}(-1,1,1,1,1) \cdot \mathbf{y}=0$ in $\mathbb{R}^{4}$, where $\mathbf{y}=$ $\left(y_{0}, y_{1}, \ldots, y_{4}\right)^{T}$ are homogeneous coordinates in $\mathbb{P}^{4}$, and a further quadric $Q: \mathbf{y}^{T} \cdot B \cdot \mathbf{y}=0$. A surface $\Phi=Q \cap S^{3}$ is called Darboux-cyclide in $S^{3}$. By a stereographic projection $S^{3} \rightarrow \mathbb{R}^{3}$ one obtains Darboux-cyclides in $\mathbb{R}^{3}$. This projection is realized by the equations

$$
x_{0}=y_{0}-y_{4}, x_{1}=y_{1}, x_{2}=y_{2}, x_{3}=y_{3} .
$$

Substituting these relations into (19), and taking $\left(y_{0}-y_{4}\right)\left(y_{0}+y_{4}\right)=y_{1}^{2}+$ $y_{2}^{2}+y_{3}^{2}$ into account, gives a quadratic equation

$$
a_{0}\left(y_{0}+y_{4}\right)^{2}-\left(y_{0}+y_{4}\right)\left(b_{1} y_{1}+b_{2} y_{2}+b_{3} y_{3}\right)+a_{1} y_{1}^{2}+a_{2} y_{2}^{2}+a_{3} y_{3}^{2}=0,
$$

representing $Q$. Thus, (19) is a Darboux cyclide. For more information on these particular algebraic surfaces one may contact [14].

We turn to paraboloids $F^{\star}$. Since $\omega \in F^{\star}$, we choose a coordinate system such that $F$ is parameterized by $\mathbf{f}(s, t)=\left(s, t, a s^{2}+b t^{2}+c\right)$, with $a b c \neq 0$. If $a$ or $b$ are zero, $F$ is a parabolic cylinder, and $\alpha(F)$ is a curve. If $c=0, F$ contains $O$. Otherwise we have $F: a x_{1}^{2}+b x_{2}^{2}+c x_{0}^{2}-x_{0} x_{3}=0$ and its dual equation reads

$$
F^{\star}:-4 a b u_{0} u_{3}+b u_{1}^{2}+a u_{2}^{2}-4 a b c u_{3}^{2}=0 .
$$

The polarity $\pi$ maps $F^{\star}$ to the quadric $\pi\left(F^{\star}\right): 4 a b x_{0} x_{3}+b x_{1}^{2}+a x_{2}^{2}-4 a b c x_{3}^{2}=$ 0 . The property $\omega \in F^{\star}$ implies $O \in \pi\left(F^{\star}\right)$. Dividing by $x_{0}$, the pedal surface

$$
G(X)=\alpha\left(F^{\star}(U)\right)=4 a b x_{3}\left(x_{1}^{2}+x_{2}^{2}+x_{3}^{2}\right)+x_{0}\left(b x_{1}^{2}+a x_{2}^{2}-4 a b c x_{3}^{2}\right)
$$

is a parabolic Darboux cyclide, an algebraic surface of degree three. If $a=b$, $F$ is a paraboloid of revolution, and if additionally $O$ coincides with the focal point of $F$, thus $c=-\frac{1}{4 a}$, the pedal surface is reducible and reads $G(X)=\left(x_{1}^{2}+x_{2}^{2}+x_{3}^{2}\right)\left(4 a x_{3}+x_{0}\right)$. The first factor $x_{1}^{2}+x_{2}^{2}+x_{3}^{2}=0$ defines the isotropic cone $\Delta$ with vertex $O$, the second factor $4 a x_{3}+x_{0}=0$ is the tangent plane at the vertex $(1,0,0,-1 /(4 a)) \mathbb{R}$ of $F$.

Theorem 9. The pedal surface $G$ of an ellipsoid or a hyperboloid $F$ is a Darboux cyclide of degree four, with $O$ as double point. The pedal surface $G$ of a paraboloid $F$ is a Darboux cyclide of degree three. In case that $O$ coincides with the focal point of a paraboloid of revolution $F$, its pedal surface is the tangent plane at $F$ 's vertex.

The family of rational offset surfaces $F_{d}$ of the quadric $F$ is mapped by $\alpha$ to the family of rational conchoid surfaces $G_{d}$ of the cyclide $G$.

Regular quadrics $F \subset \mathbb{R}^{3}$ are rational offset surfaces, see [11]. The construction is not trivial, and thus we provide an outline. Any regular quadric $F$ is the envelope of a rational one-parameter family of cones of revolution $C(u)$, with vertices at a focal conic of $F$. It is possible to parametrize these cones $C(u)$ such that the unit normal vectors of the tangent planes of $C(u)$ 
have rational norm. Since $F$ is enveloped by $C(u)$, the rationality of the norm holds for the unit normal vectors of $F$. Since the offsets of the cones $C$ are again cones of revolution $C_{d}$, the rationality of the norm holds for the unit normal vectors of the offsets $F_{d}$, too.

The pedal surface $G_{d}=\alpha\left(F_{d}^{\star}\right)$ of an offset $F_{d}^{\star}$ of a quadric $F^{\star}$, contains the pedal curves $c=\alpha(C)$ of the cones $C$. Consider a cone of revolution $C$ tangent to $F$, and let $C^{\perp}$ be the cone of revolution with vertex $O$, formed by lines perpendicular to the tangent planes of $C$. Let $S$ be the sphere with diameter $O V$, where $V$ is $C$ 's vertex. The pedal curve $c=\alpha(C)$ is the intersection $S \cap C^{\perp}$. Thus $c$ is a rational spherical curve of degree four, with double point at $O$. Finally, $G_{d}$ contains a rational one-parameter family of spherical quartic curves $c$, according to the cones of revolution $C_{d}$ enveloping $F_{d}^{\star}$.

Singular dual quadrics. The pedal construction applies also to singular dual quadrics and their offsets. Consider a singular dual quadric

$$
F^{\star}(U)=U^{T} \cdot A \cdot U, \text { with } A \in \mathbb{R}^{4 \times 4}, A=A^{T} \text { and rk } A=3,
$$

which consists of the planes through the tangent lines of a conic $c=F^{\star}$. The null-space of $A$ is the carrier plane $\gamma \supset c$. An offset surface $F_{d}^{\star}$ is a pipe surface with center curve $c$ and radius $d$. We make a short note on the degenerate cases. If rk $A=2, F^{\star}$ consists of two bundles of planes and if $\mathrm{rk} A=1$, $F^{\star}$ consists of a two-fold bundle of planes. Consider a bundle of planes with vertex $V$. According to Thales theorem, the pedal surface $G=\alpha\left(F^{\star}\right)$ is a sphere with diameter $O V$.

Assume that $c=F^{\star}$ is a conic. For studying the pedal surface $\alpha(c)$, we use the decomposition $\alpha=\sigma \circ \pi$. The polar image $Q=\pi(c)$ is a quadratic cone, with vertex $V=\pi(\gamma)$. The lines of $Q$ correspond to the pencils of planes in $c$. If $O \in \gamma, Q$ is a cylinder. If $O \in c$, a pencil of planes is mapped to the points of an ideal line $\subset \omega$, and $Q$ is a parabolic cylinder. Worth to be mentioned is the case where $Q=\pi(c)$ is a rotational cone or cylinder. This happens exactly if $O$ is contained in the focal conic $g$ of $c$. The carrier plane of $g$ is a symmetry plane of $c$, and the vertices of $g$ coincide with the focal points of $c$, and vice versa.

The inversion $\sigma$ maps a quadratic cone $Q$ to a cyclide $G=\sigma(Q)$, typically of degree four. The tangent planes $\tau(u)$ of $Q$ are mapped to spheres $S(u)=\sigma(\tau(u))$, such that $G$ is a canal surface. In case that $Q$ is a cylinder or cone of revolution, it contains also a family of inscribed spheres. Consequently, $G=\sigma(Q)$ is the envelope of two different families of spheres, thus a Dupin cyclide.

Theorem 10. The pedal surface $G=\alpha(c)$ of a conic $c$ is a Darboux cyclide, and also a canal surface. The pedal surfaces of the pipe surfaces with center curve c are conchoid surfaces $G_{d}$ of the Darboux cyclide $G$. In case that the reference point $O$ of $\alpha$ is located at the focal conic $g$ of $c$, the pedal surface $G$ is a Dupin cyclide. 
The pedal surfaces of conics $c$ are typically canal surfaces and cyclides of degree four. Degree reductions appear at first if $c$ is a parabola. Since $\omega$ touches $c, O$ is contained in $\pi(c)$, and consequently $G=\alpha(c)$ is a cubic cyclide.

Quadrics of revolution. Let the tangential cone of the reference point $O$ to the quadric $F^{\star}$ be a cone of revolution. In that case $\pi\left(F^{\star}\right)$ is a rotational quadric, the envelope of a one-parameter family of spheres. Since $\sigma$ maps spheres to spheres, $G$ is a canal surface as well. As we have already seen, this happens also if $\pi\left(F^{\star}\right)$ is a quadratic cone or cylinder, since tangent planes count as spheres. In the particular case where $\pi\left(F^{\star}\right)$ is a rotational cone or cylinder, $G$ is the envelope of two different families of spheres, thus a Dupin cyclide.

A further particular case appears when $\pi\left(F^{\star}\right)$ is a sphere. This happens if the reference point $O$ is the focal point of a rotational quadric $F^{\star}$. Consequently, $G$ is a sphere.

\subsection{Inverse pedal surfaces of quadrics}

While the previous subsection has studied quadrics as dual surfaces, we consider now quadrics $G$ and their conchoid surfaces $G_{d}$ as point sets. A quadric $G$ is represented as zero-set of a quadratic polynomial

$$
G(X)=X^{T} \cdot A \cdot X \text {, with } A \in \mathbb{R}^{4 \times 4} \text {, and } A=A^{T} .
$$

If $\operatorname{det} A \neq 0$, the quadric is regular, if rk $A=3, G$ is a quadratic cone. More degenerated cases are a pair of planes $(\mathrm{rk} A=2)$ and a two-fold plane (rk $A=1$ ). The inverse pedal surface $F^{\star}=\alpha^{\star}(G)$ of a plane $G$ is a paraboloid of revolution, with focal point $O$. For the remainder we assume rk $A=3$ or 4 .

Consider a regular quadric $G$ and assume that $G$ 's axes are parallel to the coordinate axes. Then $G$ is the zero-set of the quadratic polynomial

$$
G(X)=a_{0} x_{0}^{2}+a_{1} x_{1}^{2}+a_{2} x_{2}^{2}+a_{3} x_{3}^{2}+x_{0}\left(b_{1} x_{1}+b_{2} x_{2}+b_{3} x_{3}\right) .
$$

Applying $\alpha^{\star}$ yields $F^{\star}$, which is in fact just the dual object of (19). With the abbreviation $\mathbf{u}=\left(u_{1}, u_{2}, u_{3}\right)$, its defining polynomial reads

$$
F^{\star}(U)=u_{0}^{2}\left(a_{1} u_{1}^{2}+a_{2} u_{2}^{2}+a_{3} u_{3}^{2}\right)-u_{0} \mathbf{u}^{2}\left(b_{1} u_{1}+b_{2} u_{2}+b_{3} u_{3}\right)+a_{0} \mathbf{u}^{4} .
$$

Dual to the fact that the origin $O=(1,0,0,0) \mathbb{R}$ is a double point of $G$ in (19), the ideal plane $\omega$ is a double tangent plane of $F^{\star}$.

Quadrics $G \subset \mathbb{R}^{3}$ are rational conchoid surfaces, see [6], meaning that their conchoid surfaces $G_{d}$ allow rational polar representations $\mathbf{g}_{d}$ with rational norm $\left\|\mathbf{g}_{d}\right\|$. According to Theorem 4, rational offset parameterizations $\mathbf{f}_{d}$ of $F_{d}^{\star}$ are obtained by $\mathbf{f}_{d}=\alpha^{\star}\left(\mathbf{g}_{d}\right)$. Using the composition $\alpha^{\star}=\pi^{\star} \circ \sigma$, the surface $F^{\star}=\pi^{\star} \circ \sigma(G)$ is the dual or polar object of the cyclide $\sigma(G)$. An offset surface $F_{d}^{\star}$ is the dual or polar object of a conchoid surface $\sigma\left(G_{d}\right)$ of the cyclide $\sigma(G)$. 


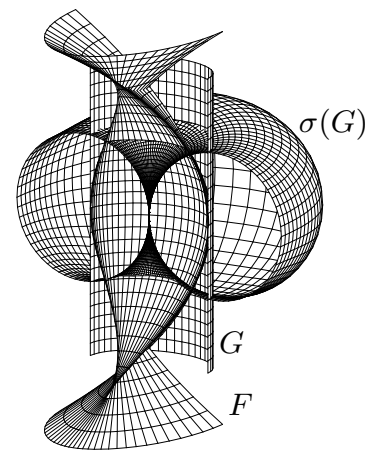

(a) Quadratic cylinder

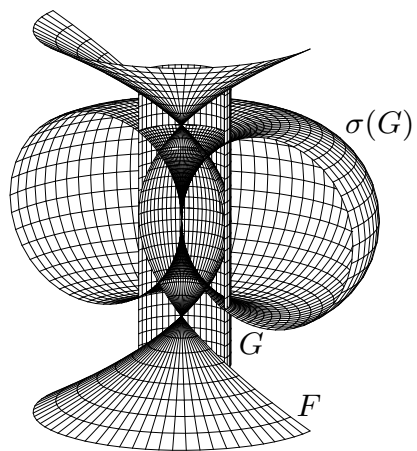

(b) Rotational cylinder

FiguRE 5. Inverse pedal surfaces of quadratic cylinders

Quadratic cone and cylinder. Let $G$ be a quadratic cone or cylinder. Since the tangent planes $\tau$ of $G$ count as spheres, the cyclide $\sigma(G)$ is a canal surface, enveloped by the spheres $S=\sigma(\tau)$ through $O$. The spheres $S$ touch $\sigma(G)$ along circles $k=\sigma(l)$, with $O \in k$. These circles correspond to the lines $l \subset G$. If $O \in \tau, \sigma(\tau)=\tau$ is a plane.

The polar image $Q=\pi^{\star}(S)=\alpha^{\star}(\tau)$ of a sphere $S$ is a quadric $Q$. Since $O \in S, Q$ is a paraboloid. Since $O \in k$ and $k \subset S$, the paraboloid $Q=\alpha^{\star}(\tau)$ touches the parabolic cylinder $P=\alpha^{\star}(l)=\pi^{\star}(k)$ along a parabola $d$. Consequently, the inverse pedal surface $F=\alpha^{\star}(G)$ of a quadratic cone $G$ is the envelope of a family of paraboloids $Q=\alpha^{\star}(\tau)$, corresponding to the tangent planes $\tau$ of $G$.

Quadratic cones and cylinders are also ruled surfaces, and thus the results from Section 4.2 apply. The paraboloids $Q$ and the parabolic cylinders $P$ touch the inverse pedal surface $\alpha^{\star}(G)=F^{\star}$ along the parabolas $d$. To understand the meaning of the parabolas $d$, we again consider the canal surface $\sigma(G)$, enveloped by spheres $S$. There exists a family of cones of revolution $D$, touching $\sigma(G)$ along the circles $k$. Since the incidences $k \subset D$ and $k \subset S$ are preserved, we have $d=\pi(D)$ or $D=\pi^{\star}(d)$.

Example. Consider the cylinder $G: x_{1}^{2} / a^{2}+x_{2}^{2} / b^{2}-x_{0}^{2}=0$. The inverse image $\sigma(G)$ is a cyclide, and $\pi^{\star}: \mathbb{P}^{3} \rightarrow \mathbb{P}^{3^{\star}}$ maps it to $\alpha^{\star}(G)=F^{\star}$, see Figure 5(a). One obtains

$$
\begin{aligned}
& \sigma(G): \frac{x_{0}^{2} x_{1}^{2}}{a^{2}}+\frac{x_{0}^{2} x_{2}^{2}}{b^{2}}=\left(x_{1}^{2}+x_{2}^{2}+x_{3}^{2}\right)^{2}, \\
& F^{\star} \quad: \quad \frac{u_{0}^{2} u_{1}^{2}}{a^{2}}+\frac{u_{0}^{2} u_{2}^{2}}{b^{2}}=\left(u_{1}^{2}+u_{2}^{2}+u_{3}^{2}\right)^{2} .
\end{aligned}
$$

The defining polynomial of $F$ is rather lengthy, and of degree eight. Even the case $a=b$ is interesting, where $\sigma(G)$ is a torus, whose meridian circles touch the $z$-axis. The inverse pedal surface is a rotational surface with the parabola $\left(-b^{2}+v^{2}, 0,2 v\right)$ as meridian curve, see Figure $5(\mathrm{~b})$. 
Sphere. Consider the sphere $G:(x-m)^{2}+y^{2}+z^{2}=r^{2}$ of radius $r$, centered at $(m, 0,0)$. Since $\sigma(G)$ is a sphere, the inverse pedal surface $F^{\star}=\pi^{\star}(\sigma(G))$ with respect to $O$ is a quadric. The defining polynomials of $G$ and $F^{\star}$ are

$$
\begin{aligned}
G(X) & =x_{1}^{2}+x_{2}^{2}+x_{3}^{2}-2 x_{0} x_{1} m+x_{0}^{2}\left(m^{2}-r^{2}\right), \\
F^{\star}(U) & =u_{0}^{2}+2 u_{0} u_{1} m+\left(u_{1}^{2}+u_{2}^{2}+u_{3}^{2}\right)\left(m^{2}-r^{2}\right) .
\end{aligned}
$$

Transforming the plane coordinates to point coordinates one obtains the known result that $F$ is a quadric of revolution, with $O$ as focal point,

$$
F: \frac{r^{2}\left(y^{2}+z^{2}\right)}{a^{2}}-\frac{x^{2}}{a}+\frac{2 m x}{a}=1 \text {, with } a=m^{2}-r^{2} .
$$

The quadric $F$ is an ellipsoid if $O$ is inside of $G$, and a hyperboloid of two sheets if $O$ is outside of $G$. Figure 6 illustrates the 2d-case. If $O \in G, F$ degenerates to a single point, according to Thales theorem. Considering the dual surface in that particular case, $F^{\star}$ is a bundle of planes, passing through that single point.

A rational polar representation $\mathbf{g}(u, v)$ of $G$ is mapped by $\alpha^{\star}$ to a rational offset parameterization $\mathbf{f}(u, v)$ of $F$. Therefore the conchoid surfaces of spheres correspond to the offset surfaces of ellipsoids and hyperboloids of revolution.

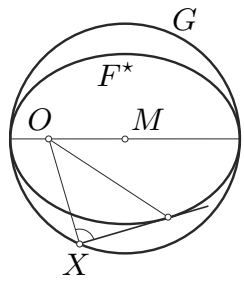

(a) Ellipse

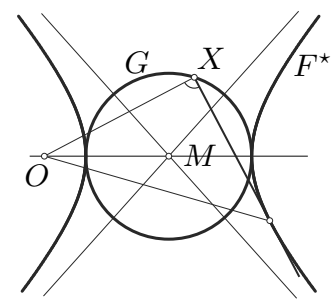

(b) Hyperbola

FiguRE 6. Inverse pedal curves $F^{\star}$ of a circle $G$

Theorem 11. The conchoid surfaces $G_{d}$ of a sphere $G$ are in birational correspondence to the offset surfaces $F_{d}$ of ellipsoids or hyperboloids of revolution $F$, depending on whether the reference point $O$ is inside or outside of $G$.

\section{Conclusion}

Relations between rational offset surfaces and rational conchoid surfaces are studied. The birational foot-point map $\alpha$ transforms a family of offset surfaces to a family of conchoid surfaces. These relations are demonstrated at hand of pedal and inverse pedal surfaces of ruled surfaces and quadrics.

There is a close relation to bisector surfaces. Considering a surface $G \subset$ $\mathbb{R}^{3}$, and a fixed reference point $O$. The bisector surface $B(G, O)$ of $G$ and $O$ 
is the envelope of symmetry planes $S$ of $O$ and a moving point $\mathbf{g} \in G$. Scaling the inverse pedal surface $F^{\star}=\alpha^{\star}(G)$ by the factor $1 / 2$ gives $B(G, O)$.

\section{Acknowledgments}

This work has been partially funded by the Spanish 'Ministerio de Economia y Competitividad' under the project MTM2011-25816-C02-01. The last two authors belong to the Research Group ASYNACS (Ref. CCEE2011/R34).

\section{References}

[1] Albano A., Roggero M., 2010. Conchoidal transform of two plane curves. Applicable Algebra in Engineering, Communication and Computing 21, 309-328.

[2] Arrondo E., Sendra J., Sendra J.R., 1997. Parametric Generalized Offsets to Hypersurfaces. Journal of Symbolic Computation 23, 267-285.

[3] Dietz R., Hoschek J., and Jüttler B., 1993. An algebraic approach to curves and surfaces on the sphere and other quadrics, Comp. Aided Geom. Design 10, 211-229.

[4] Farouki R.T., 2002. Pythagorean Hodograph curves, in: Handbook of Computer Aided Geometric Design, Farin, G., Hoschek, J., Kim, M.-S. eds.), Elsevier.

[5] Farouki, R.T., 2008. Pythagorean-Hodograph Curves: Algebra and Geometry Inseparable, Springer.

[6] Gruber D., Peternell M., 2013. Conchoid surfaces of quadrics. J. Symbolic Computation 59, 36-53.

[7] Hilton H., 1920. Plane algebraic curves. Clarendon Press.

[8] Kerrick A.H., 1959. The limacon of Pascal as a basis for computed and graphic methods of determining astronomic positions. Journal of the Institute of Navigation 6, 310-316.

[9] Loria G., 1911. Spezielle Algebraische und Transzendente Ebene Kurven: Theorie und Geschichte. Teubner, Leipzig.

[10] Krasauskas R., Peternell M., 2010. Rational offset surfaces and their modeling applications, in: IMA Volume 151: Nonlinear Computational Geometry, (eds.) I.Z. Emiris, F. Sottile, and Th. Theobald, 109-136.

[11] Peternell M., Pottmann H., 1998. A Laguerre geometric approach to rational offsets, Comp. Aided Geom. Design 15, 223-249.

[12] Peternell M., Gruber D., Sendra J., 2011. Conchoid surfaces of rational ruled surfaces, Comp. Aided Geom. Design 28, 427-435.

[13] Pottmann H., 1995. Rational Curves and Surfaces with Rational Offsets, Comp. Aided Geom. Design 12, 175-192.

[14] Pottmann H., and Shi L., and Skopenkov M., 2012. Darboux cyclides and webs from circles, Comput. Aided Geom. Design 29, 77-97.

[15] Schicho J., 2000. Proper Parametrization of Real Tubular Surfaces, J. Symbolic Computation 30, 583-593.

[16] Sendra J.R., Sendra J., 1999. Algebraic Analysis of Offsets to Hypersurfaces. Mathematische Zeitschrift 234, 697-719. 
[17] Sendra J.R., Sendra J., 2008. An algebraic analysis of conchoids to algebraic curves, Applicable Algebra in Engineering, Communication and Computing 19, 285-308.

[18] Sendra J., Sendra J.R., 2010. Rational parametrization of conchoids to algebraic curves, Applicable Algebra in Engineering, Communication and Computing $21,413-428$.

[19] Sendra J.R., Winkler F., Pérez-Diaz S., 2007. Rational Algebraic Curves: A Computer Algebra Approach. Springer-Verlag Heidelberg, in series Algorithms and Computation in Mathematics, Volume 22.

[20] Szmulowicz F., 1996. Conchoid of Nicomedes from reflections and refractions in a cone. American Journal of Physics 64, 467-471.

[21] Vršek J., Lávicka M., 2012. Exploring hypersurfaces with offset-like convolutions. Comp. Aided Geom. Design 29, 676-690.

[22] Lin W., Yu Z., Yuang E., Luk K., 2001. Conchoid of Nicomedes and Limacon of Pascal as Electrode of Static Field and a Waveguide of High Frecuency Wave. Progress In Electromagnetics Research, PIER 30, 273-284.

\author{
Martin Peternell \\ University of Technology Vienna \\ 1040 Wien \\ Austria \\ e-mail: peternell@tuwien.ac.at \\ Lukas Gotthart \\ University of Technology Vienna \\ 1040 Wien \\ Austria \\ e-mail: gotthart@geometrie.tuwien.ac.at \\ Juana Sendra \\ Dpto. Matemática Aplicada a la I.T. de Telecomunicación \\ CITSEM, Universidad Politécnica de Madrid \\ 28031Madrid \\ Spain \\ e-mail: jsendra@euitt.upm.es \\ J. Rafael Sendra \\ Dpto. de Fisica y Matemáticas \\ Universidad de Alcalá \\ 28871 Alcalá de Henares \\ Spain \\ e-mail: rafael.sendra@uah.es
}

